\title{
Global dysregulation of the chromosome 14q32 imprinted region in uterine carcinosarcoma
}

\author{
ERIC J. DEVOR ${ }^{1}$, JILLIAN N. DE MIK ${ }^{1}$, SHYAM RAMACHANDRAN ${ }^{2}$, \\ MICHAEL J. GOODHEART ${ }^{1,3}$ and KIMBERLY K. LESLIE ${ }^{1,3}$
}

\begin{abstract}
Departments of ${ }^{1}$ Obstetrics and Gynecology, and ${ }^{2}$ Pediatrics, University of Iowa Carver College of Medicine;
${ }^{3}$ Holden Comprehensive Cancer Center, University of Iowa Hospitals and Clinics, Iowa City, IA 52242, USA
\end{abstract}

Received September 30, 2011; Accepted December 20, 2011

DOI: $10.3892 /$ etm.2012.458

\begin{abstract}
Uterine carcinosarcoma (UCS) is a rare but very aggressive cancer of the female reproductive tract with an extremely poor prognosis. With the goal of understanding the role of microRNA (miRNA) dysregulation in these tumors, we profiled the expression of 667 human miRNAs in a panel of eight UCS patients and five benign control primary tissue samples. These expression profiles revealed two important characteristics of UCS. First, compared with the two most common uterine cancers, endometrial endometrioid adenocarcinoma and endometrial serous adenocarcinoma, UCS samples display a virtually unique pattern of miRNA dysregulation with an overlap of only $5 \%$ among the three tumor types. In addition, nearly one-third of the miRNAs significantly dysregulated in UCS tissues compared with benign endometrium (32 of 114) lie in a single small $(250-\mathrm{kb})$ imprinted region of chromosome 14q32. These data suggest that the presence of such a global, region-specific disruption substantially contributes to the unique histology and poor outcome of this type of cancer.
\end{abstract}

\section{Introduction}

Uterine carcinosarcoma (UCS), also known as malignant mixed Müllerian tumor (MMMT), is a rare and aggressive neoplasia characterized histologically by both carcinomatous/epithelial and sarcomatous/mesenchymal elements (1). These tumors are generally thought to account for 3-7\% of uterine cancers (2). In general, UCSs present in the late seventh decade of life, are quite advanced and have a very poor prognosis due to the aggressive nature of the tumors (3). An epidemiological study revealed that UCS and endometrial carcinoma share a similar risk factor profile including obesity and unopposed estrogen exposure (4). Moreover, the behavior of UCS more closely resembles that

Correspondence to: Dr Eric J. Devor, Department of Obstetrics and Gynecology, University of Iowa Carver College of Medicine, 375 Newton Road, 3234 MERF, Iowa City, IA 52242, USA

E-mail: eric-devor@uiowa.edu

Key words: microRNA, uterine carcinosarcoma, chromosome 14q32 of the highly aggressive endometrial carcinomas (e.g. serous adenocarcinomas) than that of other uterine sarcomas $(5,6)$. For these reasons, the 2009 revised FIGO staging for uterine sarcomas recommends that UCS be staged as a carcinoma of the endometrium (1). Collectively, these clinical data reflect a need to better understand the molecular characteristics of UCS, particularly the similarities and differences with other endometrial carcinomas.

The most contentious aspect of UCS in the past has been the origin of the tumor itself. Certain investigators have argued that UCS tumors are actually two independent tumors (the collision model), while others contend that UCS begins as a single tumor that differentiates into carcinomatous and sarcomatous elements (the monoclonal model). While the collision model has not been completely excluded, an increasing number of molecular studies support the monoclonal model. For example, patterns of X-inactivation in most UCSs are identical in the epithelial and mesenchymal components $(7,8)$. Similar findings have been reported for loss of heterozygosity (LOH), the tumor suppressor TP53, the oncogene KRAS and microsatellite instability (MSI) (8-11). Thus, the emerging consensus is that the majority of UCSs are monoclonal.

One aspect of UCS that has received little attention to date is the pattern of microRNA (miRNA) expression in these tumors compared either with benign endometrium or with other endometrial cancers. miRNAs are small (21 to $23 \mathrm{nt}$ ) regulatory RNAs whose known role in oncogenesis and cancer progression is rapidly expanding $(12,13)$. We determined the expression of 667 miRNAs in a panel of eight UCS tissue samples and compared the expression patterns with a panel of five benign endometrium, four endometrial endometrioid adenocarcinoma and four endometrial serous adenocarcinoma tissue samples. We found that the expression of 114 miRNAs was significantly dysregulated (106 overexpressed and eight underexpressed) as compared with benign endometrium, but only nine of these miRNAs were found to overlap with those significantly dysregulated in endometrial adenocarcinomas. We also found that, among the 105 significantly dysregulated miRNAs that do not overlap with the adenocarcinomas, nearly one-third (32 miRNAs) lie in the $250-\mathrm{kb}$ imprinted region in chromosome 14q32. Moreover, all but three of the 61 miRNAs in the region whose expression we measured showed at least twofold overexpression as compared with benign endometrium. 
Global dysregulation of this region in UCS is further evidenced by the altered expression of the reciprocally imprinted $\delta$-like homolog 1 (Drosophila) (DKL1)/non-coding RNA locus maternally expressed 3 (GTL2) (also known as MEG3) locus that also lies in the same chromosome 14q32 imprinted region $(14,15)$. The dysregulation of expression of such a large number of miRNAs in such a small region suggests the possibility of the disruption of a single regulatory factor, particularly in view of the evidence that the entire region is imprinted.

\section{Materials and methods}

Carcinosarcomas and benign endometrium. Primary carcinosarcoma tissues $(\mathrm{n}=8)$ and samples of benign endometrium $(n=5)$ were obtained from patients undergoing surgery in the Gynecologic Oncology Division of the Department of Obstetrics and Gynecology at the University of Iowa Hospitals and Clinics. The study was approved by the Institutional Review Board and informed consent was obtained from all patients. All carcinoma patients presented as grade 3 in stages IB to IIIC. Benign endometrium was obtained from post-menopausal patients who were cancer-free. All tissue specimens were snap-frozen and stored at $-80^{\circ} \mathrm{C}$ following histological evaluation.

RNA purification. Tissue samples were transitioned from -80 to $-20^{\circ} \mathrm{C}$ following perfusion in RNAlater ICE (Ambion, Life Technologies) according to the supplier's instructions. Total RNA was extracted from homogenized tissue using the miRvana miRNA isolation kit (Ambion, Life Technologies). RNA yield and quality were assessed on a NanoDrop M-1000 spectrophotometer and an Agilent 2100 Bioanalyzer. An RNA quality cut-off value at RIN $\geq 7.0$ was adhered to and RNAs meeting that cut-off value were standardized to $200 \mathrm{ng} / \mu \mathrm{l}$ (NanoDrop confirmed) for all subsequent analyses.

qPCR assays. miRNA expression levels were determined on miRNA TaqMan Low Density Arrays (TLDA; Applied Biosystems, Life Technologies). The A-set (v. 2.0,377 miRNAs) and the B-set (v. 2.0, 290 miRNAs) TLDA cards were used for these assays. Reverse transcription of $600 \mathrm{ng}$ of total RNA was carried out for each tissue and TLDA card using the TaqMan miRNA reverse transcription kit (Applied Biosystems, Life Technologies) and either the Human A-pool (v. 2.1) or Human B-pool (v. 2.0) Megaplex RT primers (Applied Biosystems, Life Technologies).

All 26 TLDA cards were processed on an Applied Biosystems Model 7900 Genetic Analyzer and the resulting data analyzed using Applied Biosystems StatMiner software. The expression of all 667 miRNAs in all samples was normalized against the expression of the RNU48 endogenous control RNA. Comparison of individual, normalized miRNA expression levels was performed using the $\Delta \Delta \mathrm{Ct}$ method where fold change between carcinosarcoma and benign endometrium is given as $2^{-\Delta \Delta \mathrm{Ct}}$ for each miRNA (16). Statistical significance of the fold change estimates were assessed using two-tailed t-tests with unequal variances and potential false discovery was adjusted via Bonferroni correction of the p-values. A p-value of $\leq 0.05$ was considered to indicate a statistically significant difference. $m R N A$ expression assays. Expression of the reciprocally imprinted DKL1/GTL2 locus was examined using $500 \mathrm{ng}$ of RNA from each tissue using Applied Biosystems (Life Technologies) gene-specific TaqMan Assays. RNAs were reverse-transcribed with random hexamers using the Applied Biosystems (Life Technologies) High Capacity cDNA Reverse Transcription Kit. Expression differences between carcinosarcomas and benign endometrium were calculated using the $\Delta \Delta \mathrm{Ct}$ method as above. The expressions of DLK1 and GTL2 were normalized against $18 \mathrm{~S}$ rRNA prior to analysis.

Bisulfite sequencing. To assess methylation status, bisulfite sequencing was performed on the differentially methylated region (DMR) lying between DLK1 and GTL2 in the carcinosarcomas. DNA was purified from tumor tissue and $2 \mu \mathrm{g}$ aliquots were bisulfite converted using the EZ DNA Methylation-Direct Kit (Zymo Research) according to the manufacturer's instructions. The DKL1/GTL2 DMR was then amplified from converted DNA using the following primer pair, DMRFOR: 5'-GTGGATTTGTGAGAAATGATTTTGT-3' and DMRREV: 5'-CCATTATAACCAATTACAATACCAC-3' as specified by Guens et al (15). Amplicons were cloned into pGEM T-EASY (Promega), multiple colonies were selected for each tumor and DNA was prepared using the QIAprep Spin Miniprep Kit (Qiagen). These DNAs were then sequenced in both directions on an Applied Biosystems Model 3730xl DNA Sequencer using vector M13 primers.

\section{Results}

miRNA expression. The results of the TLDA array comparisons of the eight UCS samples with the five benign endometrium samples are presented in Table I. A total of 114 miRNAs were significantly altered in the UCSs versus benign endometrium after the p-values were adjusted by Bonferroni correction to reduce the false discovery rate (FDR) (17). The majority of these differences are in the direction of overexpression among the UCSs $(n=106)$ as opposed to underexpression among the UCSs $(\mathrm{n}=8)$. A number of miRNAs, including $m i R-431$, $m i R-888$ and $m i R-206$, are very highly expressed in UCS tissues as compared with benign samples.

When compared with the $71 \mathrm{miRNAs}$ found to be significantly dysregulated among endometrial adenocarcinomas (18), only nine were found to overlap with altered UCS miRNAs (Table I). These nine miRNAs are the underexpressed $m i R-133 a$ and $l e t-7 c$ and the overexpressed $m i R-107, m i R-181 c^{*}$, miR-205, miR-210, miR-516a-3p, miR-888 and let-7f-2*.

Among the twenty most highly expressed miRNAs are $m i R-431, m i R-432, m i R-541, m i R-543$ and $m i R-369-3 p$. These five miRNAs are all from the cluster of miRNAs found in the imprinted region of chromosome 14q32, demonstrating that a quarter of the most highly expressed miRNAs in UCS are found in this small $(250 \mathrm{~kb})$ region. A complete scan of the A-set and B-set miRNAs on the TLDA arrays revealed that 43 miRNA loci in this chromosome $14 q 32$ region, accounting for a total of 61 miRNAs $(9.2 \%)$, were represented. The current miRNA content of the chromosome 14q32 cluster is 57 loci accounting for 71 miRNAs (miRBase, release 17 April 2011). Of the 61 chromosome 14q32 cluster miRNAs represented on the TLDA cards, all but three (miR-154, miR-329 and 
Table I. Fold change and adjusted p-values of the 114 microRNAs significantly dysregulated in UCSs compared with benign endometrium.

\begin{tabular}{|c|c|c|c|c|c|c|c|c|}
\hline MicroRNA & $\begin{array}{l}\text { Fold } \\
\text { change }\end{array}$ & $\begin{array}{l}\text { Adjusted } \\
\text { p-value }\end{array}$ & MicroRNA & $\begin{array}{c}\text { Fold } \\
\text { change }\end{array}$ & $\begin{array}{c}\text { Adjusted } \\
\text { p-value }\end{array}$ & MicroRNA & $\begin{array}{c}\text { Fold } \\
\text { change }\end{array}$ & $\begin{array}{c}\text { Adjusted } \\
\text { p-value }\end{array}$ \\
\hline \multicolumn{9}{|l|}{ Underexpressed } \\
\hline miR-29c & -10.6 & 0.009 & miR-143 & -11.7 & 0.009 & $\operatorname{miR}-145$ & -8.4 & 0.02 \\
\hline $\operatorname{miR}-195^{*}$ & -7.1 & 0.03 & let $-7 c$ & -7.5 & 0.03 & $\operatorname{miR}-133 \alpha$ & -8.2 & 0.04 \\
\hline let-7b & -6.2 & 0.04 & $\operatorname{miR}-29 a$ & -6.0 & 0.04 & & & \\
\hline \multicolumn{9}{|l|}{ Overexpressed } \\
\hline miR-431 & 1525.3 & 0.0001 & $m i R-888$ & 132.7 & 0.0001 & miR-216b & 91.5 & 0.0001 \\
\hline miR-543 & 65.8 & 0.0002 & miR-206 & 135.1 & 0.0003 & miR-330-5p & 46.9 & 0.0003 \\
\hline miR-15b* & 56.4 & 0.0003 & miR-153 & 30.1 & 0.0004 & $\operatorname{miR}-183$ & 43.7 & 0.0004 \\
\hline miR-33b & 30.2 & 0.0004 & let $-7 f-1^{*}$ & 79.4 & 0.0004 & miR-432 & 70.2 & 0.0007 \\
\hline miR-541 & 32.9 & 0.0008 & miR-369-3p & 67.9 & 0.0008 & miR-20b* & 92.9 & 0.001 \\
\hline miR-622 & 20.9 & 0.001 & $m i R-205$ & 26.4 & 0.002 & miR-769-3p & 86.7 & 0.002 \\
\hline miR-433 & 36.1 & 0.002 & miR-182 & 23.3 & 0.003 & $\operatorname{miR}-183^{*}$ & 26.4 & 0.003 \\
\hline miR-656 & 20.3 & 0.004 & miR-605 & 23.0 & 0.004 & miR-493* & 31.2 & 0.004 \\
\hline $\operatorname{miR}-376 a^{*}$ & 33.0 & 0.004 & $m i R-516 \alpha-3 p$ & 17.1 & 0.005 & miR-296-3p & 18.1 & 0.005 \\
\hline miR-615-3p & 53.8 & 0.006 & miR-551a & 43.7 & 0.006 & $\operatorname{miR}-192^{*}$ & 17.5 & 0.006 \\
\hline $\operatorname{miR-376b}$ & 32.6 & 0.006 & miR-892a & 58.3 & 0.006 & miR-130b* & 15.8 & 0.006 \\
\hline $\operatorname{miR}-488^{*}$ & 38.7 & 0.006 & miR-154* & 32.9 & 0.006 & miR-377 & 46.8 & 0.006 \\
\hline miR-889 & 15.4 & 0.007 & miR-496 & 40.9 & 0.007 & miR-668 & 32.8 & 0.007 \\
\hline miR-541* & 35.7 & 0.007 & miR-18a* & 12.4 & 0.008 & miR-493 & 15.4 & 0.008 \\
\hline $\operatorname{miR}-518 d-3 p$ & 14.2 & 0.008 & miR-323-3p & 13.4 & 0.009 & $\operatorname{miR}-155^{*}$ & 28.9 & 0.009 \\
\hline $\operatorname{miR}-7$ & 13.3 & 0.009 & miR-641 & 25.8 & 0.01 & miR-135b* & 17.5 & 0.01 \\
\hline $\operatorname{miR}-223^{*}$ & 12.6 & 0.01 & miR-654-5p & 17.3 & 0.01 & miR-922 & 26.7 & 0.01 \\
\hline miR-217 & 14.1 & 0.01 & $\operatorname{miR}-551 b^{*}$ & 9.7 & 0.01 & miR-539 & 13.3 & 0.01 \\
\hline $\operatorname{miR}-147 b$ & 12.8 & 0.01 & miR-499-5p & 13.7 & 0.01 & miR-382 & 18.4 & 0.01 \\
\hline miR-887 & 10.7 & 0.01 & $\operatorname{miR}-195^{*}$ & 21.1 & 0.02 & miR-639 & 11.0 & 0.02 \\
\hline $\operatorname{miR}-373$ & 22.5 & 0.02 & miR-107 & 10.0 & 0.02 & miR-582-3p & 10.2 & 0.02 \\
\hline miR-891a & 14.6 & 0.02 & miR-409-5p & 10.5 & 0.02 & $\operatorname{miR}-138-1^{*}$ & 9.6 & 0.02 \\
\hline$m i R-210$ & 8.1 & 0.02 & let $-7 f-2^{*}$ & 10.1 & 0.02 & let-7a* & 13.5 & 0.02 \\
\hline miR-651 & 10.7 & 0.02 & miR-198 & 9.8 & 0.02 & miR-346 & 8.2 & 0.02 \\
\hline $\operatorname{miR}-146 a^{*}$ & 17.5 & 0.02 & miR-937 & 12.0 & 0.02 & $\operatorname{miR}-450 b-3 p$ & 16.6 & 0.02 \\
\hline miR-505 & 9.4 & 0.02 & miR-491-3p & 18.0 & 0.02 & miR-501-3p & 13.4 & 0.02 \\
\hline $\operatorname{miR}-369-5 p$ & 8.5 & 0.02 & miR-630 & 7.6 & 0.02 & $m i R-181 c^{*}$ & 8.3 & 0.03 \\
\hline miR-18b & 8.4 & 0.03 & miR-643 & 7.6 & 0.03 & miR-506 & 15.7 & 0.03 \\
\hline miR-943 & 16.1 & 0.03 & miR-570 & 7.7 & 0.03 & miR-188-3p & 14.0 & 0.03 \\
\hline miR-299-3p & 8.7 & 0.03 & miR-941 & 8.7 & 0.03 & miR-584 & 8.5 & 0.03 \\
\hline $\operatorname{miR}-545^{*}$ & 7.9 & 0.03 & miR-638 & 8.0 & 0.04 & miR-409-3p & 9.6 & 0.04 \\
\hline miR-875-5p & 7.5 & 0.04 & miR-485-3p & 8.9 & 0.04 & miR-379* & 7.5 & 0.04 \\
\hline miR-561 & 7.2 & 0.04 & miR-661 & 7.2 & 0.04 & miR-432* & 7.1 & 0.04 \\
\hline miR-223 & 7.0 & 0.04 & miR-487b & 8.6 & 0.05 & miR-301b & 8.2 & 0.05 \\
\hline miR-127-5p & 6.7 & 0.05 & miR-654-3p & 7.4 & 0.05 & miR-376a & 7.2 & 0.05 \\
\hline miR-372 & 6.8 & 0.05 & & & & & & \\
\hline
\end{tabular}

MicroRNAs in the chromosome 14q32 imprinted cluster are shown in bold type. MicroRNAs shared between UCSs and endometrioid adenocarcinomas are italicized. UCS, uterine carcinosarcoma.

$m i R$-412) were overexpressed in the UCSs by at least two-fold and, of these, 32 achieved statistical significance (Fig. 1). Thus, while chromosome $14 \mathrm{q} 32$ miRNAs represent $9.2 \%$ of the miRNAs present on the TLDA arrays, they account for a statistically significant $30.5 \%\left(\chi^{2}=56.9, \mathrm{p}<0.001\right)$ proportion of the significantly overexpressed miRNAs. Moreover, among 


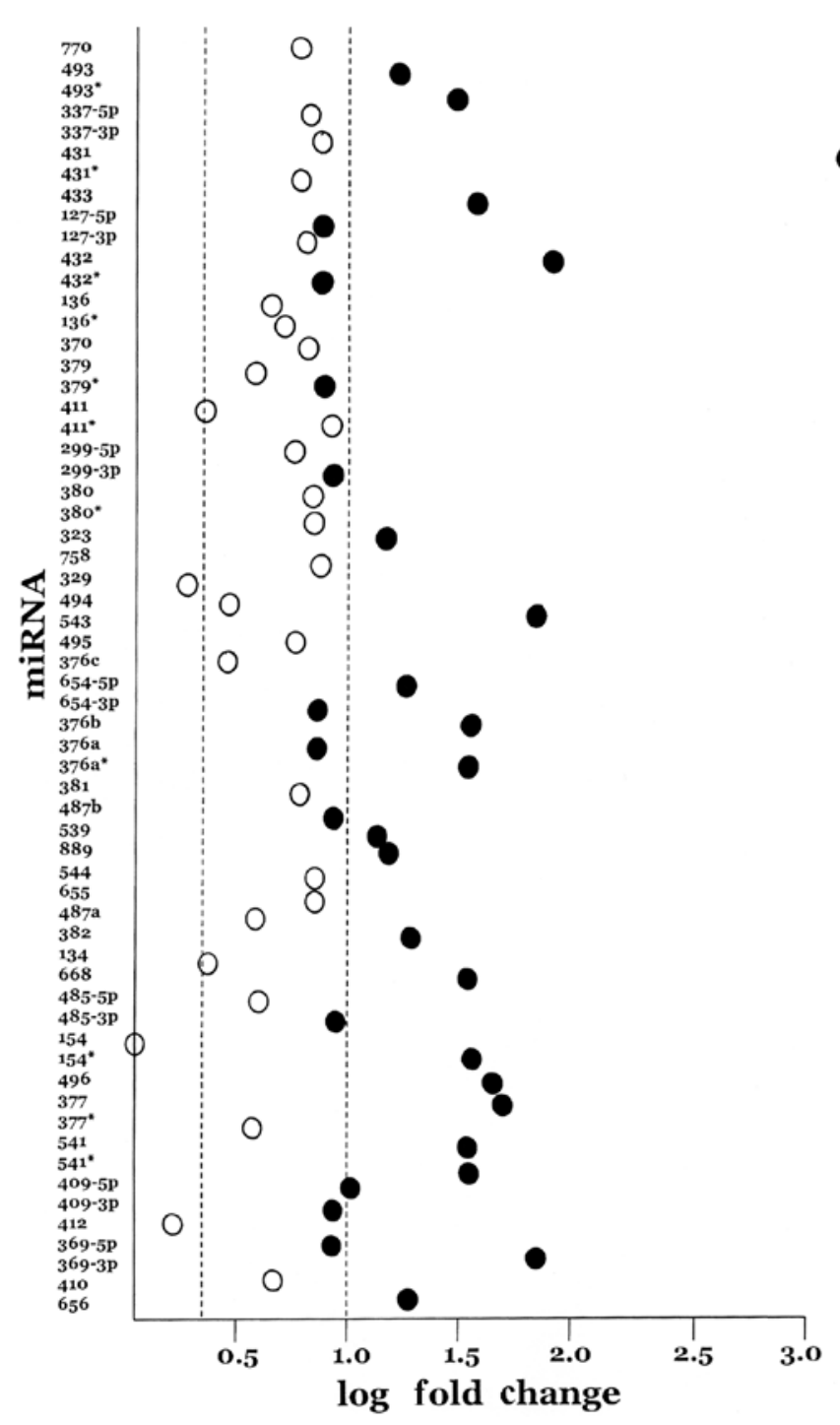

Figure 1. Log fold change of 61 microRNAs in the chromosome 14q32 imprinted region. The microRNAs are shown in their physical order from $5^{\prime}(m i R-770)$ to $3^{\prime}(m i R-656)$. The dashed lines indicate 2 -fold and 10 -fold increased expression in UCSs compared with benign endometrium. Closed circles represent those microRNAs reaching at least $\mathrm{p} \leq 0.05$ statistical significance and open circles indicate those microRNAs at $\mathrm{p}>0.05$.

the 18 miRNAs in which mature sequences from both sides of the hairpin are expressed, that is, both the $5 p$ and the $3 p$ arms, eight show nearly equal expression.

DLK1/GTL2 expression in UCS. On the centromeric flank of the chromosome $14 \mathrm{q} 32$ miRNA cluster is the reciprocally imprinted locus composed of DLK1 and GTL2. In order to discover whether the global dysregulation of the miRNAs in chromosome 14q32 extends to other genes, the expression of both DKL1 and GTL2 was assessed in the UCS tumor panel as well as the benign endometrium group. DKL1/GTL2 expression in UCSs and benign endometrium, normalized against 18S rRNA transcription, is shown in Fig. 2. These data show that GTL2 expression is higher (lower $\Delta \mathrm{Ct}$ ) than DLK1 expression (higher $\Delta \mathrm{Ct}$ ) in the benign endometrium panel whereas levels are nearly equal in the UCS panel. In a reciprocally imprinted locus a modest negative correlation of expression levels of the two transcripts is anticipated. In the

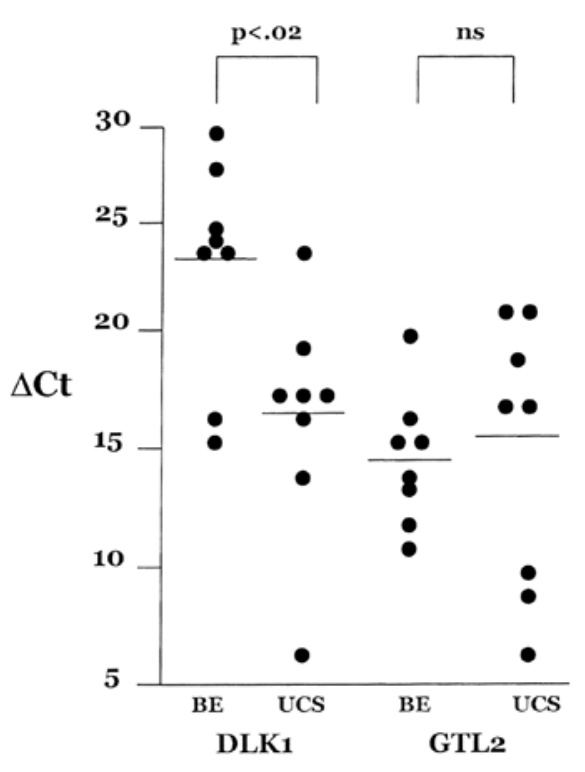

Figure 2. Expression of DLK1 and GTL2 mRNA in uterine carcinosarcoma (UCS) and in benign endometrium (BE). Three additional benign samples were added to the panel to equalize sample sizes. DKL1 shows a significant increase in expression in UCS compared with benign endometrium while GTL2 shows no change in expression. ns, not significant.

benign endometrium panel, the correlation between DLK1 and GTL2 is $r=-0.21$ [not significant (ns)] whereas we see a positive correlation in the UCS panel, $\mathrm{r}=0.52$ (ns). While GTL2 expression levels were not altered in the UCSs as compared to benign endometrium, the DLK1 expression level increased 92 -fold $(\mathrm{p}<0.02)$ in the UCSs. This result suggests that paternal chromosome expression (DLK1) has become uncoupled from maternal chromosome expression (GTL2).

Previous studies have shown that reciprocal expression of DKL1 and GTL2 is regulated in part by the methylation status of a DMR located between the two loci (14). Bisulfite sequencing of the DKL1/GTL2 DMR in the eight carcinosarcomas and five benign endometrium samples revealed no change in methylation patterns. This implies that the change in expression of DLK1 in UCS tissues versus benign endometrium is not attributable to altered methylation.

\section{Discussion}

UCSs display a unique miRNA expression signature. A recent TLDA-based miRNA expression survey of uterine cancers including a small number of UCSs concluded that these tumors display a unique miRNA dysregulation signature compared with other uterine cancers, specifically endometrial adenocarcinomas (19). Our data reinforce and expand upon this observation. Specifically, we show from our larger panel of miRNAs that, among the same 667 miRNAs surveyed by us in both endometrial adenocarcinoma (18) and UCS, only nine of the 71 miRNAs significantly dysregulated in endometrial adenocarcinoma compared with benign endometrium overlap with the 114 miRNAs significantly dysregulated in UCS compared with benign endometrium. Apart from these few miRNAs, it is the unique miRNA signature of UCSs that dominates the data presented here. A recent TLDA-based miRNA study of UCSs focused upon 
the biphasic nature of these tumors (20). Using the A-set miRNAs, the present study separated the epithelial from the mesenchymal components of 23 tumors and reported on a modest differential miRNA expression signature observed in the two components. Not surprisingly, a number of the miRNAs reported in that study also appear in the present study. Among these is $m i R-29 c$ which is more highly expressed in the epithelial component but significantly underexpressed overall compared with benign endometrium. This miRNA has been shown to be involved in the regulation of the extracellular matrix marker SPARC and other markers associated with invasion and metastasis (21). Another miRNA shown to be differentially expressed in UCSs and underexpressed overall in UCSs compared with benign endometrium is $m i R-23 b$ which is also negatively associated with the proliferation of cancer cells (22). One notable finding is that none of the five members of the miR-200 family, known to be intimately involved in epithelial to mesenchymal transition (19,23-25) and significantly overexpressed in the epithelial component of UCSs (20) as well as in endometrial adenocarcinomas (18), achieved statistical significance in our data, although all five $m i R-200$ family members were more highly expressed in UCSs than in benign endometrium.

miRNAs in the chromosome 14q32 imprinted region are globally dysregulated. In the above-mentioned study on miRNA expression in the epithelial and mesenchymal components of UCSs (20), 24 miRNAs were shown to be more highly expressed in the mesenchymal component than in the epithelial component. Of these, seven $(29.2 \%)$ are from the imprinted region at chromosome $14 \mathrm{q} 32$. This is a statistically significant over-representation of the chromosome $14 \mathrm{q} 32$ imprinted region miRNAs present on the A-set TLDA card $\left(\chi^{2}=7.7, p<0.05\right)$ and is consistent with the statistically significant overrepresentation of miRNAs from this region in UCSs compared with benign endometrium that we report here. Our data also demonstrate that the dysregulation of miRNA expression among the UCSs is distributed across the entire $251 \mathrm{~kb}$ breadth of the region. However, the extent of this phenomenon is clearly contained within the boundaries of the imprinted region as three miRNAs at the 5' boundary, $m i R-342-5 p, m i R-342-3 p$ and $m i R-345$, and the miRNA closest to the 3 ' boundary, $m i R-203$, are all expressed at the same level in the UCSs as in the benign endometrium. Future studies are necessary to understand the mechanisms by which miRNA expression in this region is coordinately regulated and dysregulated.

The correlated expression of miRNAs in the chromosome $14 q 32$ imprinted region has previously been reported. For example, correlated overexpression was observed in acute myeloid leukemia (26) and in gastrointestinal stromal tumors without loss of chromosome 14q (27). Cytogenetic effects, either amplifications or deletions, are not at issue here as no such events have ever been reported on chromosome 14 in uterine cancers $(28,29)$.

miRNAs in the chromosome 14q32 imprinted cluster show uncharacteristically low complementarity with cellular mRNA targets, suggesting that they are involved in a shared mechanism of action involving general inhibition of translation rather than degradation of specific mRNA targets (27). In mice, coordinated expression of the entire cluster has been linked with a DMR between the imprinted non-miRNA loci DLK1/GTL2 (30). Our examination of the methylation status of this DMR in relation to DLK1/GTL2 expression in our UCSs and benign endometrium clearly shows that this cis-acting regulation is disrupted, and it is likely that this regulatory disruption extends throughout the region and results in the observed miRNA expression patterns as well.

We have carried out an miRNA expression survey of UCSs which confirms that these tumors display a largely unique miRNA signature. Most striking is the observation that the entire imprinted region at chromosome $14 q 32$ is dysregulated with all but one of the 61 miRNAs assayed overexpressed in UCSs compared with benign endometrium and more than half (32 of 60) reaching statistical significance. We find that this dysregulation includes the reciprocally imprinted gene pair DLK1/GTL2 lying at the upstream boundary of the region where, in spite of a 'normal' methylation pattern in the intergenic DMR, both members of the pair are highly expressed. It is possible that this DMR regulates expression of the entire region in cis (30). Coordinated overexpression of such a large block of miRNAs may contribute to the unique nature and aggressive behavior of these tumors. In addition, the significant increase in DLK1 expression may also play a role, as DLK1 has been shown to inhibit Notch signaling in mesenchymal multipotent cells (31). Whatever the effect of increased DLK1 expression coupled with the global overexpression of the chromosome 14q32 miRNA cluster may turn out to be, understanding the mechanism that leads to the dysregulation of multiple miRNAs at once may present a unique opportunity to identify a therapeutic target whose effects are widespread.

\section{Acknowledgements}

This study was supported by NIH Grant RO1CA99908 to K.K.L. and in part by the Department of Obstetrics and Gynecology Research Development Fund. We thank the University of Iowa Carver College of Medicine DNA Core Facility, particularly Garry Hauser and Mary Boes, for their invaluable assistance and Dr Kristina W. Thiel for assistance in manuscript preparation.

\section{References}

1. D'Angelo E and Prat J: Uterine sarcomas: A review. Gynecol Oncol 116: 131-139, 2010.

2. Evans HL, Chawla SP, Simpson C and Finn KP: Smooth muscle neoplasms of the uterus other than ordinary leiomyoma. A study of 46 cases with emphasis on diagnostic criteria and prognostic factors. Cancer 62: 2239-2247, 1988.

3. Kernochan LE and Garcia RL: Carcinosarcomas (malignant mixed Müllerian tumor) of the uterus: Advances in elucidation of biologic and clinical characteristics. J Natl Compr Canc Netw 7: 550-557, 2009

4. Zelmanowicz A, Hildesheim A, Sherman ME, et al: Evidence for a common etiology for endometrial carcinomas and malignant mixed mullerian tumors. Gynecol Oncol 69: 253-257, 1998.

5. Silverberg SG, Major FJ, Blessing JA, et al: Carcinosarcoma (malignant mixed mesodermal tumor) of the uterus. A Gynecologic Oncology Group pathologic study of 203 cases. Int J Gynecol Pathol 9: 1-19, 1990.

6. Sreenan JJ and Hart WR: A pathologic study of 29 metastatic tumors: Further evidence for the dominant role of the epithelial component and the conversion theory of histogenesis. Am J Surg Pathol 19: 666-674, 1995. 
7. Jin Z, Ogata S, Tamura G, et al: Carcinosarcomas (malignant mullerian mixed tumors) of the uterus and ovary: A genetic study with special reference to histogenesis. Int J Gynecol Pathol 22: 368-373, 2003.

8. Wada H, Enomoto T, Fujita M, et al: Molecular evidence that most but not all carcinosarcomas of the uterus are combination tumors. Cancer Res 57: 5379-5385, 1997.

9. Abeln EC, Smit VT, Wessels JW, et al: Molecular genetic evidence for the conversion hypothesis of the origin of malignant mixed Müllerian tumors. J Pathol 183: 424-431, 1997.

10. Fujii H, Yoshida M, Gong ZX, et al: Frequent genetic heterogeneity in the clonal evolution of gynecological carcinosarcoma and its influence on phenotypic diversity. Cancer Res 60: 114-120, 2000.

11. Taylor NP, Zighelboim I, Huettner PC, et al: DNA mismatch repair and TP53 defects are early events in uterine carcinosarcoma tumorigenesis. Mod Pathol 19: 1333-1338, 2006.

12. Melo SA and Esteller M: Dysregulation of microRNAs in cancer: Playing with fire. FEBS Lett 585: 2087-2099, 2011.

13. Rovira C, Güida MC and Cayota A: MicroRNAs and other small silencing RNAs in cancer. IUBMB Life 62: 859-868, 2010.

14. Kircher M, Bock C and Paulsen M: Structural conservation versus functional divergence of maternally expressed microRNAs in the Dlk1/Gtl2 imprinting region. BMC Genomics 9: 346, 2008.

15. Guens E, De Temmerman N, Hilven P, et al: Methylation analysis of the intergenic differentially methylated region of DLK1-GTL2 in human. Eur J Hum Genet 15: 352-361, 2007.

16. Livak KJ and Schmittgen TD: Analysis of relative gene expression data using real-time quantitative PCR and the 2(-delta delta C(T)) method. Methods 25: 402-408, 2001.

17. Guo W, Sarkar SK and Peddada SD: Controlling false discoveries in multidimensional directional decisions, with applications to gene expression data on ordered categories. Biometrics 66 : 485-492, 2010.

18. Devor EJ, Hovey AM, Goodheart MJ, Ramachandran S and Leslie KK: microRNA expression profiling of endometrial endometrioid adenocarcinomas and serous adenocarcinomas reveals profiles containing shared, unique and differentiating groups of microRNAs. Oncol Rep 26: 995-1002, 2011.

19. Ratner ES, Tuck D, Richter C, et al: MicroRNA signatures differentiate uterine cancer tumor subtypes. Gynecol Oncol 118 251-257, 2010.

20. Castilla MA, Moreno-Bueno G, Romero-Perez L, et al: Micro-RNA signature of the epithelial-mesenchymal transition in endometrial carcinosarcoma. J Pathol 223: 72-80, 2011.
21. Sengupta S, den Boon JA, Chen IH, et al: MicroRNA 29c is down-regulated in nasalpharyngeal carcinomas, up-regulating mRNAs encoding extracellular matrix proteins. Proc Natl Acad Sci USA 105: 5874-5878, 2008.

22. Salvi A, Sabelli C, Moncini S, et al: MicroRNA-23b mediates urokinase and c-met downmodulation and a decreased migration of human hepatocellular carcinoma cells. FEBS J 276: 2966-2982, 2009.

23. Cochrane DR, Spoelstra NS, Howe EN, Nordeen SK and Richer JK: MicroRNA-200c mitigates invasiveness and restores sensitivity to microtubule-targeting chemotherapeutic agents. Mol Cancer Ther 8: 1055-1066, 2009.

24. Gregory P, Bert A, Paterson E, et al: The miR-200 family and miR-205 regulate epithelial to mesenchymal transition by targeting ZEB1 and SIP1. Nat Cell Biol 10: 593-601, 2008.

25. Vrba L, Jensen TJ, Garbe, JC, et al: Role for DNA methylation in the regulation of miR-200c and miR-141 expression in normal and cancer cells. PLoS ONE 5: e8697, 2010.

26. Dixon-McIver A, East P, Mein CA, et al: Distinctive patterns of microRNA expression associated with karyotype in acute myeloid leulaemia. PLoS ONE 3: e2141, 2008.

27. Haller F, von Heydebreck A, Zhang JD, et al: Localization- and mutation-dependent microRNA (miRNA) expression signatures in gastrointestinal stromal tumours (GISTs), with a cluster of co-expressed miRNAs located at 14q32.31. J Pathol 220: 71-86, 2010.

28. Micci F, Tiexeira MR, Haugom L, Abeler VM and Heim S: Genomic aberrations in carcinomas of the uterine corpus. Genes Chrom Cancer 40: 229-246, 2004.

29. Salvesen HB, Carter SL, Mannelqvist M, et al: Integrated genomic profiling of endometrial carcinoma associates aggressive tumors with indicators of PI3 kinase activation. Proc Natl Acad Sci USA 106: 4834-4839, 2009.

30. Seitz H, Royo H, Bortolin M-L, et al: A large imprinted microRNA gene cluster at the mouse Dlk1-Gt12 domain. Genome Res 14: 1741-1748, 2004.

31. Sánchez-Solana B, Nueda ML, Ruvira MD, et al: The EGF-like proteins DLK1 and DLK2 function as inhibitory non-canonical ligands of NOTCH1 receptor that modulate each other's activities. Biochim Biophys Acta 1813: 1153-1164, 2011. 